\title{
CBT: making effective plans
}

\section{David Osborne \& Chris Williams}

\begin{abstract}
SUMMARY
Working with people who are experiencing mental health difficulties brings many challenges about how best to progress towards shared goals. The cognitive-behavioural therapy (CBT) approach can provide a structure and framework for effective treatment. It aims to help people identify goals and work towards them. We describe a practical approach that provides a structure for step-by-step planning, engaging the patient and promoting planned change.

\section{DECLARATION OF INTEREST}

C.W. is author of various CBT books and online resources for which he receives royalties, and is director of a company that commercialises these resources.
\end{abstract}

In cognitive-behavioural therapy (CBT), planning starts by writing a goal on a piece of paper and committing to trying to achieve it. The choice of what to do first is influenced by a range of complex factors. For example, the patient's and practitioner's understanding of the problems, strategies they have used before, personal strengths and weaknesses, and the resources available to them, such as other people or having the stability of a job and income. A decision needs to be made about what is most achievable, as well as identifying potential problems that may derail the plan.

However a clinician decides to help their patient make plans, the most important factor in making it effective is that it is made collaboratively. The goal needs to actively involve the patient, and not be defined by the clinician alone. With this in mind, we have outlined a handy three-step approach - Plan, Do and Review - with the first step, Plan, integrating this spirit of collaboration (Williams 2010).

\section{Step 1: Make a plan}

It's important to start with small goals based on something that's important to the patient. Discussion could cover the top four or five things they would like to change. Of these, select one that you both think is achievable and relevant to the patient's problems. To inform that choice consider a target that is big enough to move things forward, but small enough that it doesn't seem impossible or too anxiety-provoking.

Larger or general goals can be broken down into constituent parts, steps or chunks; select one of those parts to start things off. It is helpful to make the goal specific and measurable, so that you and your patient know how far they've come towards achieving it.

Write down the plan. A good first plan might involve a small behavioural change. Examples include doing something that they rate highly for enjoyment, or that develops a sense of closeness to others or that gives a feeling of achievement (or all three at the same time). Ideally, what they choose should also fit their values and how they would like to live their life. Early successes make it more likely that your patient will feel good and stay motivated, so encourage them to choose something that will make a positive difference.

The plan should identify:

- what they will do

- when they will do it

- any potential blocks or difficulties that may arise.

This allows a problem-solving stance to be taken even before the plan is put into action. For example, how could the patient modify things to adapt their plan if it rained that day, or their child was ill? A planner sheet (Fig. 1) aids writing a specific goal.

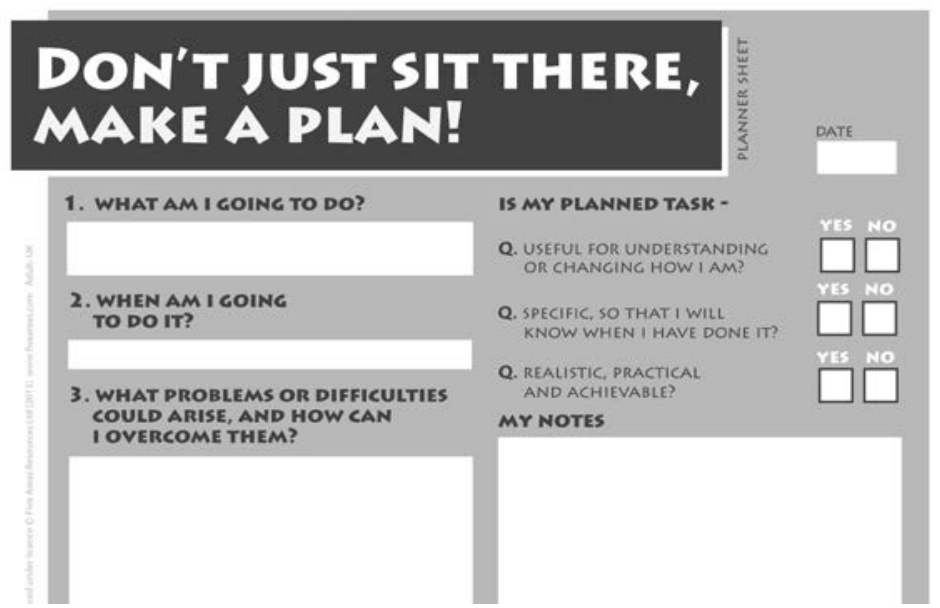

Planner sheet (৫) 2015 Five Areas Ltd, available free from www.llttf.com and reproduced with permission).
David Osborne has a Certificate of Completion of Training (CCT) in medical psychotherapy and has psychiatry in NHS Greater Glasgow and Clyde. Chris Williams is Professor of Psychosocial Psychiatry at the University of Glasgow and President-elect of the British Association for Behavioural and Cognitive Psychotherapies. He researches models of low-intensity CBT delivery in clinical and community settings. He is author of the popular Living Life to the Full web course (www.llttf.com). Correspondence Professor Chris Williams, Institute of Health and Wellbeing, Administration Building GRH, University of Glasgow, Glasgow G12 OXH. UK. Email: chris. williams@glasgow.ac.uk

'This is the second in a series of refreshments on aspects of cognitive-behavioural therapy. The first (Colvin 2015) considered the formulation. The next will address mindfulness. begun training in general adult 


\section{OK, HOW DID IT GO?}

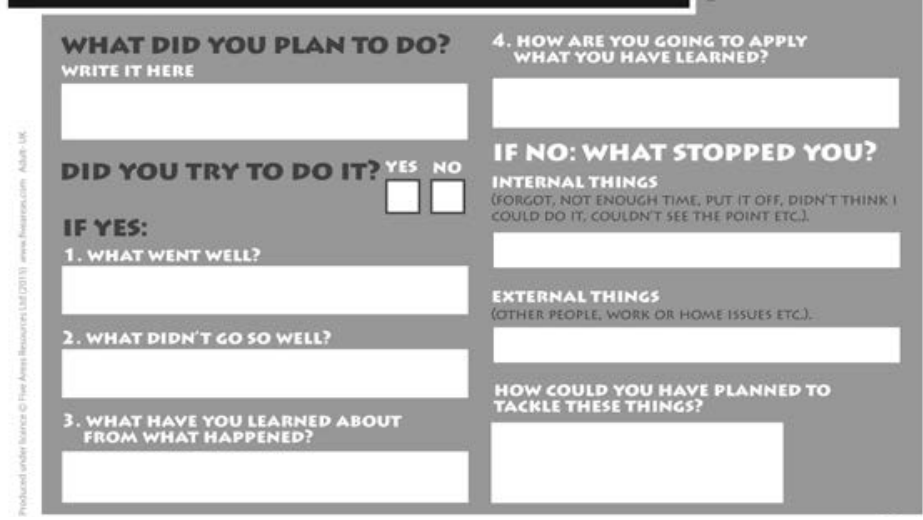

Review sheet (C) 2015 Five Areas Ltd, available free from www.llttf.com and reproduced with permission). is where the review stage becomes particularly useful in understanding exactly what happened (e.g. they forgot to do it, talked themselves out of it, or perhaps an unexpected external event occurred that made it hard to stick to the plan). Asking what they think went well first, followed by a joint discussion about what could have been done better, provides a constructive and structured approach to feedback. This aspect is worth slowing down, as significant learning can occur that can be applied to the next plan. Linking progress back to the initial CBT formulation (Williams 2002b; Colvin 2015) helps see progress in the light of the wider treatment plan. A review sheet (Fig. 2) promotes learning.

You can find free copies of the planner and review worksheets at the Living Life to the Full website (www.Llttf.com).

\section{Monitoring progress and moving forward}

Rating scales can help monitor progress. Short-, medium- and long-term goals can then be used to plan a sequence of plans that aim to move the person towards their long-term goal.

\section{Acknowledgements}

The Plan, Do, Review model is used with permission of Five Areas Ltd.

\section{References and further reading}

Colvin L, Williams C (2015) CBT: helping work out why you feel as you do (formulation). BJPsych Advances, 21: 405-6.

Williams C, Garland A (2002a) Identifying and challenging unhelpful thinking. Advances in Psychiatric Treatment, 8: 377-86.

Williams C, Garland A (2002b) A cognitive-behavioural therapy assessment model for use in everyday clinical practice. Advances in Psychiatric Treatment, 8: 172-9.

Williams C, Chellingsworth M (2010) CBT: A Clinician's Guide to Using the Five Areas Approach. CRC Press. 